\title{
Nonequilibrium Relaxations and Aging Effects in a Two-Dimensional Coulomb Glass
}

\author{
J. Jaroszyński and Dragana Popoviđ买 \\ National High Magnetic Field Laboratory, Florida State University, Tallahassee, Florida 32310
}

(Dated: June 28, 2018)

\begin{abstract}
The relaxations of conductivity have been studied in the glassy regime of a strongly disordered two-dimensional electron system in Si after a temporary change of carrier density during the waiting time $t_{w}$. Two types of response have been observed: a) monotonic, where relaxations exhibit aging, i.e. dependence on history, determined by $t_{w}$ and temperature; b) nonmonotonic, where a memory of the sample history is lost. The conditions that separate the two regimes have been also determined.

PACS numbers: 71.55.Jv, 71.27.+a, 71.30.+h
\end{abstract}

The dynamics of glasses and other systems out of equilibrium is one of the most challenging and rapidly evolving topics in condensed matter research [1]. Although glassy behavior may dominate the low-temperature properties of many complex materials near quantum phase transitions [2], such as the metal-insulator transition (MIT), quantum glasses are less understood than their classical counterparts. Experimental studies of charge or Coulomb glasses [3], which are of particular relevance to the MIT, have been scarce [4, 5, [6], and limited to insulating systems far from the MIT. Recent observations [7, 8, 9] of glassiness in a two-dimensional electron system (2DES) in Si open up opportunities for exploring glassy phenomena over a wide range of all the relevant parameters. The carrier density $n_{s}$, for example, can be varied continuously on the same sample from the deep metallic into the deep insulating regime. The onset of glassiness was, in fact, found [7, 8, 9] to take place at a density $n_{g}>n_{c}\left(n_{c}\right.$ - the critical density for the MIT), i.e. on the metallic side of the MIT, consistent with theory [10].

The manifestations of glassiness in a 2DES for $n_{s}<n_{g}$, as demonstrated by resistance noise measurements [7, 8], include a dramatic slowing down of the electron dynamics and correlated statistics consistent with the hierarchical picture of glasses. Furthermore, studies of the relaxations of the conductivity $\sigma$ [9], following a rapid change of $n_{s}$, show that the equilibration time $\tau_{e q}$ [11] diverges exponentially as temperature $T \rightarrow 0$, suggesting a glass transition at $T_{g}=0$. However, even at $T$ that are not too low (e.g. $\sim 1 \mathrm{~K}), \tau_{e q}$ exceeds easily not only the experimental time window but also the age of the Universe. This makes it relatively easy to study the out-of-equilibrium relaxation of $\sigma$ at times $t \ll \tau_{e q}$, where one expects to find properties common to other types of glasses. Indeed, for $t \ll \tau_{e q}$, the relaxations obey [9] $\sigma\left(t, T, n_{s}\right) / \sigma_{0}\left(T, n_{s}\right) \propto$ $t^{-\alpha\left(n_{s}\right)} \exp \left[-\left(t / \tau\left(T, n_{s}\right)\right)^{\beta\left(n_{s}\right)}\right]$ with $\tau$ diverging as $T \rightarrow 0$ $\left(\sigma_{0}\left(T, n_{s}\right)\right.$ - equilibrium $\sigma$ at a given $T$ and $n_{s} ; 0<$ $\left.\alpha\left(n_{s}\right)<0.4,0.2<\beta\left(n_{s}\right)<0.45\right)$. Such scaling has been observed in spin glasses above $T_{g}[12]$. The dependence $\tau \propto \exp \left(\gamma n_{s}^{1 / 2}\right)(\gamma-$ a proportionality constant $)[9]$ is a strong indication for the relevance of Coulomb interactions between 2D electrons [13] in the $n_{s}<n_{g}$ regime.
The most puzzling feature of the relaxation in Ref. [9], however, is that the system equilibrates only after it first goes farther away from equilibrium. This "overshooting" (OS) of $\sigma_{0}$ is discussed in more detail below.

A key characteristic of relaxing glassy systems is the loss of time translation invariance, reflected in aging effects [15, 16]. The system is said to exhibit aging if its response to an external excitation depends on the system history in addition to the time $t$. Some history dependence was observed in a 2DES [7], but there have been no systematic and detailed studies. Here we report the first such study, where, for a given $n_{s}, \sigma(t)$ was measured after the temporary change of $n_{s}$ during the waiting time $t_{w}$. The measurement history was varied systematically by changing $t_{w}$ and $T$. Two types of response have been observed: a) monotonic, for relatively "small" excitations, where relaxations depend on $t_{w}$, i.e. the 2DES exhibits aging; b) nonmonotonic, for sufficiently "large" excitations, where $\sigma(t)$ overshoots $\sigma_{0}$ and relaxations no longer depend on $t_{w}$ (memory loss). The conditions that separate the two regimes have been identified and discussed.

Measurements were carried out on a 2DES in Si metaloxide-semiconductor field-effect transistors with a $50 \mathrm{~nm}$ oxide thickness, and a relatively large amount of disorder (the $4.2 \mathrm{~K}$ peak mobility $\approx 0.06 \mathrm{~m}^{2} / \mathrm{Vs}$ with the substrate (back-gate) bias [14] $V_{\text {sub }}=-2 \mathrm{~V}$ ). The sample length $\times$ width were $1 \times 90 \mu \mathrm{m}^{2}$ (sample A) and $2 \times 50 \mu \mathrm{m}^{2}$ (sample B). The same devices were used in Ref. [9], and they were also very similar to the samples used in noise studies [7]. The density $n_{s}$ is controlled by the gate voltage $V_{g}$, such that $n_{s}\left(10^{11} \mathrm{~cm}^{-2}\right)=4.31\left(V_{g}[\mathrm{~V}]-V_{T}\right)$, where the threshold voltage for forming the 2D layer $V_{T}=6.3 \mathrm{~V}$ for $V_{\text {sub }}=-2 \mathrm{~V} ; n_{g}\left(10^{11} \mathrm{~cm}^{-2}\right) \approx 7.5$ and $n_{c}\left(10^{11} \mathrm{~cm}^{-2}\right) \approx 4.5[9]$. The samples and the standard ac lock-in technique (typically $13 \mathrm{~Hz}$ ) that was used to measure $\sigma$ have been described in more detail elsewhere [7].

The experimental protocol [Figs. 1(a) and 1(b)] starts with the 2DES, induced by an applied gate voltage $V_{0}$, in equilibrium at $10 \mathrm{~K}$. The sample is then cooled to the measurement temperature $T$. Unlike glasses with $T_{g} \neq 0$, this does not result in any visible relaxations on our experimental time scales, and $\sigma_{0}\left(V_{0}, T\right)$ is thus established. $V_{g}$ is then switched rapidly (within $1 \mathrm{~s}$ ) to a different 


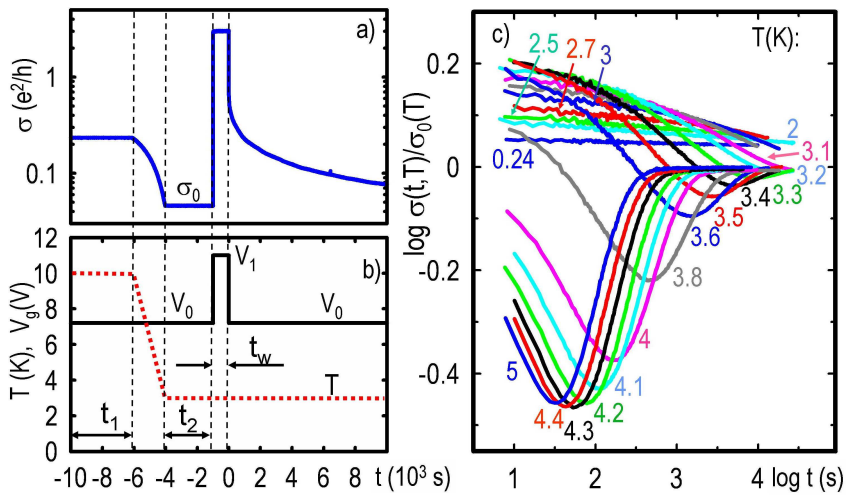

FIG. 1: (Color online) (a) Sample A. $\sigma(t)$ for $V_{0}=7.2 \mathrm{~V}$ $\left[n_{s}\left(10^{11} \mathrm{~cm}^{-2}\right)=3.88<n_{c}\right], V_{1}=11 \mathrm{~V}\left[n_{s}\left(10^{11} \mathrm{~cm}^{-2}\right)=\right.$ 20.26], $t_{w}=1000 \mathrm{~s}$, and $T=3.0 \mathrm{~K}$. (b) The corresponding experimental protocol: $V_{g}(t)$ and $T(t)$. The results do not depend on the cooling time (varied from 30 minutes to 10 hours), nor on $t_{1}$ and $t_{2}$ (varied from 5 minutes to 8 hours each). (c) Sample B. $\sigma(t)$ for $V_{0}=8 \mathrm{~V}\left[n_{s}\left(10^{11} \mathrm{~cm}^{-2}\right)=\right.$ $\left.7.33 \lesssim n_{g}\right], V_{1}=11 \mathrm{~V}, t_{w}=1000 \mathrm{~s}$, and several $T$, as shown. $T=2.3,1.8,1.3,1.0,0.75,0.5 \mathrm{~K}$ data were omitted for clarity.

value $V_{1}$, where it is kept for a time $t_{w}$. Finally, it is changed back (within $1 \mathrm{~s}$ ) to $V_{0}$, and the slowly evolving $\sigma\left(t, V_{0}, T\right)$ is measured. We note that $t=0$ is defined as the time when $V_{g}$ reattains its original value $V_{0}$. An analogous protocol has been used often in studies of other glassy materials [5]. In general, we expect the larger values of $t_{w}$ or $T$ to correspond to stronger excitations of the 2DES, because the system will have more time or more thermal energy, respectively, to wander away from its original equilibrium state during the time $t_{w}$.

Figure 1(c) shows some relaxations measured for a fixed $t_{w}$ at different $T$ for a given $V_{0}$ and $V_{1}$. The data are not shown for the first few seconds (comparable to our sampling time $\sim 1 \mathrm{~s}$ ), when $\sigma$ changes rapidly, consistent with the $\sigma_{0}\left(V_{g}\right)$ dependence [14]. In general, the observed behavior is very complicated, but it can be divided roughly into two types. At higher $T, \sigma(t)$ first goes below $\sigma_{0}$, and then even farther away from equilibrium. Eventually, it changes "direction" and approaches $\sigma_{0}$. Similar nonmonotonic behavior was observed after exciting 2DES out of equilibrium by a rapid change of $n_{s}$ [9]. At lower $T$, on the other hand, there is no OS of equilibrium and $\sigma$ approaches $\sigma_{0}$ monotonically, similar to observations in other electron glasses [5].

$\sigma(t)$ were also measured for different $t_{w}$ at a fixed $T$ and given $V_{0}$ and $V_{1}$. Some typical data, including those for two different $V_{0}$, are shown in Fig. 2. In general, the waiting time $t_{w}$ has a dramatic effect on the relaxations, both qualitatively (OS vs. no OS) and quantitatively (e.g. more than two orders of magnitude difference in $\sigma(t)$ in Fig. 2(d)), including instances of OS when $t_{w^{-}}$ dependence vanishes [Fig. 2(b)]. $\sigma(t)$ were also investigated after all the electrons have been removed from the
$2 \mathrm{D}$ layer during the time $t_{w}$. In a 2DES in Si, unlike other electron glasses, this may be accomplished simply by reducing $V_{g}$ by a sufficient amount. If the slow dynamics is dominated by intrinsically glassy behavior of the interacting electrons rather than by their response to extrinsic slow degrees of freedom (e.g. motion of nearby impurities, although exponentially unlikely at our $T$ [14]), one expects that $\sigma(t)$, measured after the electrons are reintroduced with the initial $V_{g}=V_{0}$, will not depend on $t_{w}$. The measurements confirm this expectation [Fig. 2(f)], providing further support for the glassiness in the 2DES.

A detailed analysis of $\sigma(t)$ in the case of OS [e.g. highest $T$ curves in Fig. 1(c)] finds the behavior very similar to that reported in Ref. [9]. Therefore, we do not show the fits to the data, but instead summarize the main results. For example, at $t$ just before the minimum in $\sigma(t)$ [17], $\sigma\left(t, T, n_{s}\right) / \sigma_{0}\left(T, n_{s}\right) \propto \exp \left[-\left(t / \tau\left(T, n_{s}\right)\right)^{\beta\left(n_{s}\right)}\right]$, with $\tau$ and $\beta$ [18] similar to those in Ref. 9]. After the minimum in $\sigma(t)$, the approach to equilibrium at the longest $t$ is described by a simple exponential process $\sigma / \sigma_{0}=\left[1-A(T) \exp \left(-t / \tau_{e a}\right)\right]$ with $1 / \tau_{e q} \propto \exp \left(-E_{A} / T\right)$ $\left(E_{A} \approx 57 \mathrm{~K}\right.$ as before [9, 11]). The main difference between the equilibrations that follow the two experimental protocols is that the prefactor $A$ did not depend on $T$ in Ref. [9], whereas here $A(T)$ decreases with decreasing $T$ and vanishes at a finite $T$ (Fig. 3 (a) inset). This, at least partly, reflects the observation that, in Ref. [9], the minima in the $\sigma(t)$ curves only move to lower values of $\sigma$ and to later $t$ with decreasing $T$, whereas in the current protocol, the different $T$ curves at $t$ after the minimum [Fig. 1(c)] are no longer parallel to each other, the minima become "shallower", and eventually vanish at a finite $T$. The latter is shown in Fig. 3(a), where the $T$-dependences of the amplitude of the relaxations at the minima, defined as $\left[\log \left(\sigma / \sigma_{0}\right)\right]_{\min }$, are plotted for different $t_{w}$. The amplitudes extrapolate to zero at $T$ that are in a good agreement with those obtained from $A(T)$. Thus the data seem to indicate that here the OS does not simply fall out of our experimental time window at low $T$ as in Ref. 9] but rather that it vanishes at a finite $T$. The $\sigma(t)$ curves at lower $T$ support this conclusion: their monotonic approach to $\sigma_{0}$ [from above in Fig. 1(c)] is consistent with a power-law form at the shortest $t$ (lowest $T$ ) and, at the longest $t$, with a simple exponential process with the same $E_{A} \approx 57 \mathrm{~K}$.

The procedure shown in Fig. 3(a) was applied to all the data in order to determine the values of $\left(T, t_{w}\right)$ where OS disappears. The results [Fig. 3(b)] clearly divide the phase space in two, such that the OS is not observed for smaller values of $\left(T, t_{w}\right)$ (e.g. at $1 \mathrm{~K}$, not even at $t \simeq 2 \times 10^{5} \mathrm{~s}$ ). This confirms our earlier speculation [9] that the OS occurs for sufficiently strong perturbations out of equilibrium. We note that the relevant time (energy) scale is given by $\tau_{e q}(T)$ [Fig. 3(b)]. In order to understand this result better, we discuss the experiment in more detail. During $t_{w}$, after $V_{g}$ is switched rapidly 

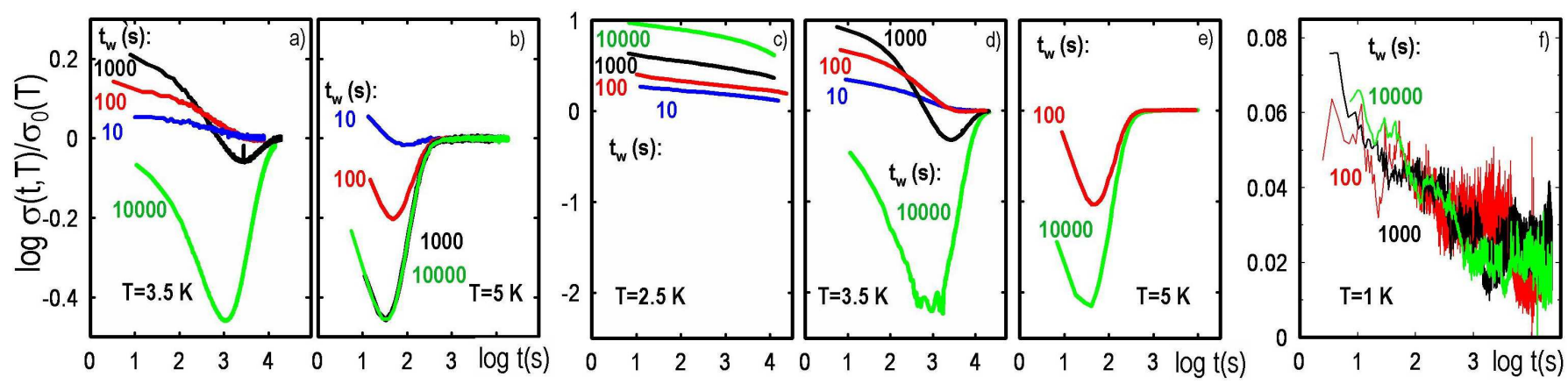

FIG. 2: (Color online) $\sigma(t)$ for different $t_{w}$ at fixed $T$, as shown. (a)-(b) Sample B; $V_{0}=8$ V, $V_{1}=11$ V; (c)-(e) Sample B; $V_{0}=7.2 \mathrm{~V}, V_{1}=11 \mathrm{~V}$. (f) Sample A; $V_{0}=7.2 \mathrm{~V}$ and $V_{1}=6 \mathrm{~V}\left(n_{s}=0\right)<V_{T}=6.3 \mathrm{~V}$, i.e. all electrons are removed from the $2 \mathrm{D}$ layer during $t_{w}$.
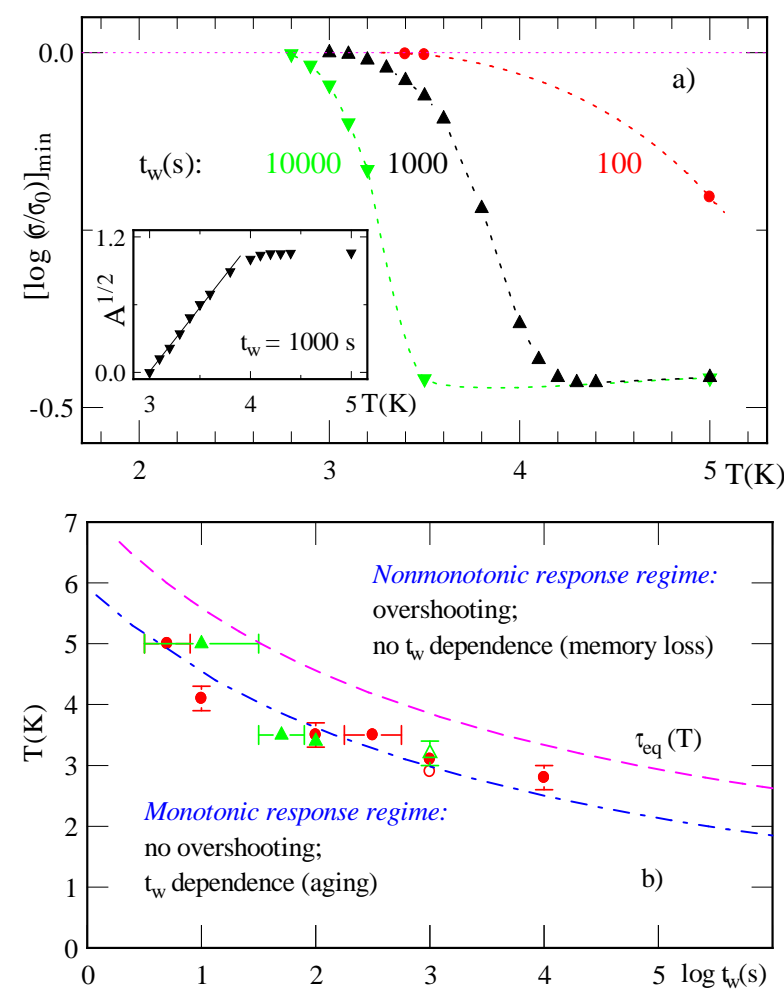

FIG. 3: (Color online) (a) $\log \left(\sigma / \sigma_{0}\right)$ at the $\sigma(t)$ minima (data with overshooting) vs. $T$ for different $t_{w}$. Sample B; $V_{0}=$ $8 \mathrm{~V}, V_{1}=11 \mathrm{~V}$. The lines guide the eye. Inset: $A(T)$ for $t_{w}=1000 \mathrm{~s}$. The solid line is a linear fit. (b) The symbols (solid and open from devices B and A, respectively) show the values of $\left(T, t_{w}\right)$ where the overshooting vanishes. Red dots and green triangles correspond to $V_{0}=8 \mathrm{~V}, V_{1}=11 \mathrm{~V}$, and $V_{0}=7.2 \mathrm{~V}, V_{1}=11 \mathrm{~V}$, respectively. The blue dot-dashed line guides the eye. The purple dashed line represents $\tau_{e q}(T)$.

from $V_{0}$ to $V_{1}, \sigma\left(t, V_{1}, T\right)$ evolves with $t$ as described in Ref. [9]. Some small amplitude relaxations are still visible [albeit not on the scale of Fig. 1(a)] even at the highest $V_{1}=11 \mathrm{~V}$ where $n_{s}>n_{g}$, but $\sigma_{0}$ is still so small that $k_{F} l<1\left(k_{F}-\right.$ Fermi wave vector, $l-$ mean free path) [19].
If the 2DES equilibrates at a new state corresponding to $V_{1}$, i.e. if $\tau_{e q} \ll t_{w}\left(\tau_{e q}\right.$ does not depend on $\left.n_{s}[9]\right)$, then the rest of this protocol, namely the switching of $V_{1}$ back to $V_{0}$ and the subsequent relaxation, are equivalent to the protocol employed in Ref. [9]. Hence both here and in Ref. 9], the OS is observed only when the 2DES is excited out of thermal equilibrium. Of course, if $\tau_{e q} \ll t_{w}$, then any further increase in $t_{w}$ will have no effect on $\sigma(t)$, as indeed observed [Fig. 2(b)]. On the other hand, when $t_{w} \ll \tau_{e q}$ [monotonic response regime in Fig. 3(b)], $\sigma(t)$ clearly depend on history (aging effect), i.e. on $t_{w}$ [see e.g. Fig. [2(c)] during which the system relaxes away from its initial equilibrium state determined by $V_{0}$ and towards a new equilibrium state determined by $V_{1}$. It can be also said that the system has a memory of the time it spent with $V_{g}=V_{1}$. This is very similar to spin glasses, for example, where $T$ or a magnetic field play a role of $n_{s}$ in our experiment. Finally, in the crossover regime $t_{w} \lesssim \tau_{e q}$ [a region between the two dashed lines in Fig. 3(b)], both some OS and $t_{w}$-dependence are observed [Fig. 2(d)].

The nonmonotonic response of $\sigma$, which occurs when the system is taken out of thermal equilibrium, has been observed for all applied $1 \leq\left|\Delta V_{g}\right|=\left|V_{1}-V_{0}\right|(\mathrm{V}) \leq 4.7$ $\left(\left|\Delta n_{s}\right| / n_{s} \approx 20-100 \%\right)$. Since, by reducing $n_{s}$, the 2DES goes from a metallic regime, with no metastable states, to a glassy, and then glassy insulating regime, it seems reasonable to assume that the density of metastable states increases as $n_{s}$ is reduced by a large amount, reflecting the increasing complexity of the free energy landscape. The 2DES are also known to be relatively poorly coupled thermally to the surrounding lattice, especially in $\mathrm{Si}$, and thermal equilibrium at very low $\mathrm{T}$ (below $\sim 1 \mathrm{~K}$ ) is achieved mainly by conduction through the contacts and the leads. This and the available data suggest that the rapid change of $V_{g}$ is an approximately adiabatic process. In that case, the reduction of $n_{s}$, accompanied by an increase in the density of metastable states, would lead to the cooling of the 2DES. As a result, $\sigma$ would decrease with $t(d \sigma / d T>0$ in the regime of interest) below $\sigma_{0}(T)$. This initial cooling of the 2DES and the eventual 
warm-up to the bath $T$ would give rise to a minimum in $\sigma(t)$ as observed. Likewise, a nearly adiabatic increase of $n_{s}$ would lead to the heating of the 2DES, resulting in a maximum in $\sigma(t)$ [17]. The time-scale for equilibration would be determined both by heat leaks from the bath and by slow processes [20] related to collective rearrangements of the 2DES to adjust to the new conditions. The applied $\left|\Delta V_{g}\right|$ are expected to trigger major rearrangements of the electron configuration since the corresponding shifts of the Fermi energy $\Delta E_{F} \gg T$ [13, 21]. This situation, coupled with possibly substantial changes in the screening of the 2DES across the MIT [22], is fundamentally different from the cooling of the 2DES at a fixed $n_{s}$ when $\Delta T \ll E_{F}$. Charge rearrangements have been also invoked to interpret the nonmonotonic relaxation in insulating granular metals 23]. The cooling (heating) scenario proposed here is consistent with the data but other interpretations may be possible, especially since OS is found in many systems, ranging from manganites 24] to biological systems [25]. For example, OS may be a general feature of systems that are far from equilibrium [26].

The adiabatic cooling (heating) effects should not be relevant for $t_{w} \ll \tau_{e q}$, i.e. for "small" excitations, since the final state will have a large configurational similarity with the original state due to the shortness of $t_{w}$. Such small perturbations of a Coulomb glass are hence expected to lead to memory effects [27], all of which are consistent with our results. Studies of other electron glasses, where no OS but aging and memory effects were seen [5], were done in such a perturbative regime. An equivalent (deep in the $t_{w} \ll \tau_{e q}$ limit) study in the 2DES would be of great interest, especially across the MIT, but that is beyond the scope of this paper.

Finally, we have attempted to determine the microscopic origin of the activation energy $E_{A} \approx 57 \mathrm{~K}$ by repeating some of the measurements for different $V_{s u b}$. In addition to changing the disorder, $V_{s u b}$ moves the position of the $2 \mathrm{D}$ subband with respect to the bottom of the Si conduction band, and it also affects the splitting between the subbands of the 2DES [14, 28]. If the exponential process at long $t$ results from an activation to $\mathrm{Si}-\mathrm{SiO}_{2}$ interface traps or to an upper subband, it can be estimated [7, 14] that the applied range $-5 \leq V_{\text {sub }}(\mathrm{V}) \leq 0$ will have a significant impact on the value of $E_{A}$. We find, however, no change in $E_{A}$, which seems to rule out the above two processes as mechanisms for equilibration.

We have demonstrated that the 2DES in Si exhibits aging effects, one of the hallmarks of glassy behavior, and identified precisely the conditions that lead to memory loss and nonmonotonic response. The observed complex dynamics of the electronic transport is strikingly similar to that of other systems that are far from equilibrium.

We are grateful to I. Raičević for technical assistance, V. Dobrosavljević for useful discussions, NSF DMR0403491 and NHMFL via NSF Cooperative Agreement DMR-0084173 for financial support. D. P. also acknowl- edges the hospitality of the Aspen Center for Physics.

Electronic address: dragana@magnet.fsu.edu

[1] See Slow Relaxations and Nonequilibrium Dynamics in Condensed Matter, edited by J.-L. Barrat, M. Feigelman, J. Kurchan, and J. Dalibard, (Springer, Berlin, 2003).

[2] E. Miranda et al., Rep. Prog. Phys. 68, 1 (2005).

[3] J. H. Davies et al., Phys. Rev. Lett. 49, 758 (1982); M. Grünewald et al., J. Phys. C 15, L1153 (1982); M. Pollak et al., Sol. Energy Mater. 8, 81 (1982); J. H. Davies et al., Phys. Rev. B 29, 4260 (1984); M. Pollak, Philos. Mag. B 50, 265 (1984).

[4] D. Monroe et al., Phys. Rev. Lett. 59, 1148 (1987).

[5] M. Ben-Chorin et al., Phys. Rev. B 48, 15025 (1993); A. Vaknin et al., Phys. Rev. Lett. 81, 669 (1998); Phys. Rev. Lett. 84, 3402 (2000); Phys. Rev. B 65, 134208 (2002); V. Orlyanchik et al., Phys. Rev. Lett. 92, 066801 (2004).

[6] G. Martinez-Arizala et al., Phys. Rev. Lett. 78, 1130 (1997); Phys. Rev. B 57, R670 (1998); E. Bielejec et al., Phys. Rev. Lett. 87, 256601 (2001); L. M. Hernandez et al., Phys. Rev. Lett. 91, 126801 (2003); T. Grenet, Eur. Phys. J. B 32, 275 (2003); M. Lee et al., J. Phys. Condens. Matter 17, L439 (2005).

[7] S. Bogdanovich and D. Popović, Phys. Rev. Lett. 88, 236401 (2002).

[8] J. Jaroszyński et al., Phys. Rev. Lett. 89, 276401 (2002); Phys. Rev. Lett. 92, 226403 (2004).

[9] J. Jaroszyński et al., Phys. Rev. Lett. 96, 037403 (2006).

[10] V. Dobrosavljević, D. Tanasković, and A. A. Pastor, Phys. Rev. Lett. 90, 016402 (2003).

[11] In Ref. [9], this time was denoted by $\tau_{\text {high }} / k_{1}=(3.7 \times$ $\left.10^{-4} \mathrm{~s}\right) \exp \left(E_{A} / T\right)\left(E_{A} \approx 57 \mathrm{~K}\right.$ independent of $\left.n_{s}\right)$.

[12] C. Pappas et al., Phys. Rev. B 68, 054431 (2003).

[13] In 2D: $E_{F} / U \propto n_{s}^{1 / 2}\left(E_{F}-\right.$ Fermi energy, $U$ - Coulomb energy); $E_{F}[\mathrm{~K}]=7.31 n_{s}\left[10^{11} \mathrm{~cm}^{-2}\right]$ for electrons in Si 14$]$.

[14] T. Ando et al., Rev. Mod. Phys. 54, 437 (1982).

[15] L. C. E. Struik, Physical Aging in Amorphous Polymers and Other Materials (Elsevier, Amsterdam, 1978).

[16] See Complex Behavior of Glassy Systems, edited by M. Rubí and C. Pérez-Vicente (Springer, Berlin, 1997).

[17] For $V_{0}>V_{1}$, the OS appears as a maximum in $\sigma(t)$, in analogy with Ref. [9]. Switching the values of $V_{0}$ and $V_{1}$ gives a different maximal $\left|\log \left(\sigma / \sigma_{0}\right)\right|$.

[18] $\tau$ and $\beta$ depend only on the final $n_{s}$, not on $\left(V_{1}-V_{0}\right)$.

[19] Drude $\sigma=g_{v}\left(e^{2} / h\right)\left(k_{F} l\right) ; g_{v}=2$ is a valley degeneracy. None of the effects described here are seen for $k_{F} l>1$.

[20] This is analogous to "internal heat leaks" in nuclear magnetic refrigeration. See F. Pobell, Matter and Methods at Low Temperatures (Springer-Verlag, Berlin, 1992).

[21] M. Müller et al., J. Phys. IV France 131, 167 (2005).

[22] A. A. Pastor et al., Phys. Rev. Lett. 83, 4642 (1999); M. Müller et al., Phys. Rev. Lett. 93, 256403 (2004); S. Pankov et al., Phys. Rev. Lett. 94, 046402 (2005).

[23] N. Kurzweil et al., Phys. Rev. B 75, 020202(R) (2007).

[24] P. Levy et al., Phys. Rev. Lett. 89, 137001 (2002).

[25] P. Nelson, Biological Physics: Energy, Information, Life (W. H. Freeman and Company, 2003), Chapter 12.

[26] See H. Morita et al., Phys. Rev. Lett. 94, 087203 (2005).

[27] E. Lebanon et al., Phys. Rev. B 72, 174202 (2005). 
[28] X. G. Feng et al., Phys. Rev. Lett. 83, 368 (1999). 